\title{
Analysis of the Relationship between Various Microorganisms in Insects
}

\author{
Jialin Guo \\ Luohe Medical College, Luohe, Henan, 462000
}

Keywords: Bacteria; symbiotic system; bacterial substitution; pathogens; biological control

\begin{abstract}
Insects can infect different types of symbiotic microorganisms at certain developmental stages. These commensal organisms play an important role in the process of host growth, reproduction, adaptation to the environment and evolution. A large number of studies have found that different symbiotic microbes in insects do not exist and function in isolation, but have some connections to each other to varying degrees. In this paper, focusing on commensal bacteria, this paper reviews the relationship between different symbiotic bacteria in the past 10 years and the relationship between symbiotic bacteria and other insect-related microorganisms (pathogenic bacteria, fungi and viruses, and insect symbiotic fungi). Among them, the focus is on the coexistence of different bacteria in insects and the cooperation of host functions, the bacterial substitution that has occurred or may occur in insects, and the influence of symbiotic bacteria on the pathogen sensitivity of the host. Finally, the research prospects in this field are discussed in the context of the extensive application of various biological techniques in the study of symbiotic bacteria.
\end{abstract}

\section{Introduction}

A variety of microorganisms, such as bacteria, fungi, protozoa, etc., are commonly found in insects, and species that are symbiotic with insects are of concern because they can affect certain important life processes of insects. In the case of commensal bacteria, they can be divided into two major categories, primary and secondary, depending on the distribution characteristics, mode of transmission, function, and evolutionary relationship with insects. Native symbiotic bacteria have been present in the host for a considerable period of time, usually in special bacteriocytes, using vertical propagation (from the mother to the offspring), and the co-evolution with the host is obvious, the survival of the host Indispensable; secondary symbiotic bacteria infect insects relatively late, can be distributed in cells (such as bacteria, fat cells) or outside the cells (such as the intestinal wall, hemolymph) ), both vertical and horizontal, and the degree of co-evolution with the host is low, not necessary for the survival of the host. Symbiotic bacteria (especially native species) can play an important role in the growth, reproduction, survival and adaptation of insects, such as helping the host digest food and providing the host with essential nutrients (such as amino acids, fatty acids, vitamins, etc.). It regulates host reproduction and enhances the host's resistance to external biotic or abiotic stress factors (such as pathogens, parasitic wasps, high temperatures, insecticides, etc.). Due to its multiple functions, symbiotic bacteria and their relationship with the host have become an important research field of entomology. This research can not only provide a theory for explaining the adaptability of insects, population dynamics, insect diversity formation and evolutionary mechanisms. Based on the basis, it can also provide ideas for exploring new technologies for pest biological control. Different degrees of action can occur between different microorganisms in an insect. Focusing on commensal bacteria, this paper reviews recent research progress over the past 10 years, mainly reflecting the interaction between different commensal bacteria and commensal bacteria and insect pathogens and symbiotic fungi, in order to enhance our overall research dynamics in this field. understanding.

\section{Relationship between different symbiotic bacteria}

Among the many symbiotic bacteria of insects, some species coexist in the same tissue or cells 
of the host (such as bacteria-containing cells), and some even "parasitize" in another bacteria, which propagate vertically together. For example, in Bemisia tabaci (Gennadius), Trialeurodes vaporariorum (Westwood) and Acyrthosiphon pisum (Harris), secondary bacteria Hamiltonella and Wolbachia, Arsenophonus sp., Rickett The corpuscle Rickettsiella is present together in the bacterium-containing cells; in the citrus whitefly Planococcus citri (Risso), the $\gamma$-proteobacteria Moranella endobia is located in a $\beta$-proteobacteria Tremblada princeps; in the genus Cicadella viridis (L. Among them, a Sodalis-like bacterium is located in another symbiotic bacteria, Sulcia muelleri. These special ways of existence of bacteria encourage people to think and explore: what functions do different bacteria living together have, and whether there is some interaction between them. After extensive research, people have found synergy, substitution or inhibition between different symbiotic bacteria in various insects.

When a bacterium (secondary species) invades an insect that has been infected with another bacterium (native or secondary), the two bacteria undergo a synergistic evolution to form two distinct relationships: one is that both become The symbiosis necessary for the host, coexisting friendly, and forming a certain degree of cooperation and dependence on the function of the host; the other is that the bacterial genome that originally existed changed dramatically and eventually disappeared from the host, and later Invasive bacteria dominate and gradually replace the original bacteria. The synergistic phenomenon of different symbiotic bacteria is mainly found in Homoptera insects, including spider mites, mites, mites, whiteflies, hibiscus, aphids, etc. These insects feed on the sap of the plant xylem or phloem and depend on symbiosis. Bacteria provide some organic nutrients (such as amino acids, etc.) that are necessary for growth and development but are lacking in the diet. Studies have found that many of these insects are infected with two or more commensal bacteria, one of which is mostly native bacteria (eg, spider mites, mites, mites, etc.) are Sulcia muelleri, whitefly and hibiscus. Among them are Tremblaya and Carsonella, and the others are secondary bacteria. These two types of bacteria show strong complementarity in biosynthesis and metabolism, and together provide the necessary nutrients for the host. For example, the S. muelleri study found that The bacterium was infected with head lice in the Permian 260 million years ago.

Suborder insects, still present in many species of this subspecies and genetically highly conserved; in the genus Homalodisca vitripennis (Germar), the bacterium and a gamma-proteobacteria Baumannia cicadellinicola coexist in the bacterium-containing cells, B. cicadellinicola has a very limited ability to synthesize amino acids (only two can be synthesized), mainly providing vitamins and coenzymes to the host, while S. muelleri can synthesize most amino acids (8 species) necessary for the host, but cannot synthesize vitamins and coenzymes. There is a subtle collaborative relationship between the functions of the host. There is another interaction between bacteria in the Homoptera, that is, a substance that a symbiotic bacterium needs to provide life activities for another bacterium, and it is dependent on it. For example, in the pea mites, Hamiltonella defensa (protecting the host against parasitic wasps in hemipteran insects such as aphids) cannot synthesize eight essential amino acids by themselves. These amino acids are mainly composed of aphid native bacteria Buchnera.

provide. In the citrus meal, the genome of the $\beta$-proteobacteria T. princeps has been significantly degraded, and some key genes related to protein translation are lost. The related proteins, metabolites and tRNAs required for the synthesis of amino acids are located in the bacterium. Provided by the gamma-proteobacteria $M$. endobia, the two exhibit a typical dependence. Collaboration and interdependence of different commensal bacteria are also present in other insects, such as species that rely on commensal bacteria to digest and utilize food (such as termites), and species that do not have access to all the nutrients needed from food (such as tsetse flies). Among them, the situation in tsetse flies is quite special and is considered to be the "early" type of interaction between bacteria. On the one hand, the two bacteria Wigglesworthia (native, present in the bacteria-containing cells) and Sodalis (secondary, mainly in the intestine) have developed a synergistic and dependent tendency: Wigglesworthia can synthesize the vitamins necessary for the host and Sodalis. B1, Sodalis cannot synthesize vitamin B1 but retains a set of genes encoding the vitamin B1 transport system to ensure that vitamin B1 is obtained from the environment to meet its 
proliferation and intracellular infection processes; on the other hand, the genomes of the two bacteria There is a clear overlap in composition, and the Sodalis genome contains most of the genes of Wigglesworthia, indicating that the two have not yet established a true collaborative relationship with the function of the host.

The ability to form stable cooperative relationships between different commensal bacteria depends on many factors, such as the genotype of each different bacteria, the function and mode of action of the host, the ability to utilize the space and nutrient resources in the host, and the genotype of the host. The occurrence of factors such as host plants and natural enemies of host insects in the external environment, and the formation of cooperative relationships among bacteria are the result of adaptive evolution under the combined effects of these factors. Since most commensal bacteria cannot be isolated and cultured in vitro, it is difficult to study their molecular biology and biochemical characteristics in depth, so there is little understanding of the molecular basis of collaboration among different bacteria. Studies have shown that there are gene exchange phenomena between different symbiotic bacteria, thereby promoting the formation of interdependence. In coexisting bacteria, many common genes (redundant genes) are gradually lost, leading to the simplification or degradation of the genome, and finally the biosynthetic pathways of different bacteria are in a state of little overlap, thus forming a collaboration or dependence. In the future, it is necessary to conduct in-depth research on the mutual coordination and regulation mechanism of interaction bacteria in proliferation, gene expression, genome reduction and evolution.

As early as the early 1960s, the substitution of symbiotic bacteria was found in the larvae. In the past 10 years, relevant reports have gradually increased, and are found in insects such as aphids, mites, weevils, termites, mites, and flies. The most typical of these is the replacement of native bacteria in aphids and elephants. For example, in some species of Cerataphidini, the native Buchnera has been replaced by extracellular yeast, and the bacteria-containing cells no longer exist. In the scorpion Adelges spp., its ancestors were infected with the $\beta$-proteobacteria Vallotia and the $\gamma$-proteobacteria Ecksteinia/Gillettellia 65 million years ago. Vallotia in some of the scorpions was Steffania sometime 60 million years ago. Instead, Ecksteinia/Gillettellia, another ball python, was replaced by Profftia between 40 and 60 million years ago.

Among the elephants, Nardonella is the oldest native bacterium, at least 125 million years old. In some species of the genus Sitophilus and the genus Curculio, the bacterium has been completely replaced by bacteria such as Sodalis.

In some insects, there is a tendency for primary bacteria to be replaced by secondary bacteria (Table 1). The most direct evidence comes from the study of aphids. For example, when the native Buchnera of the pea cockroach is artificially removed, the pea aphid secondary symbiont (PASS) can invade the bacterium containing cells originally occupied by Buchnera and provide the host with some essential amino acids and other nutrients; In the coexisting state, PASS can also inhibit Buchnera at a certain period of host reproduction, thereby affecting the growth and reproduction of the host, which shows that PASS has the possibility of replacing Buchnera through competition. In another example, in the Cinara cedri Mimeur, although Buchnera still exists, most of the metabolic functions have been lost, including the ability to synthesize the essential tryptophan and riboflavin in the host, while the other bacteria, Serratia symbiotica.

(The former is in the bacteria-containing cells) can make up for these functions; at the same time, the existence of these two bacteria is very special, the proportion of space occupied by S. symbiotica in the bacteria-containing cells is close to or slightly larger than Buchnera, which is obviously different. In other aphids (S. symbiotica is significantly less than Buchnera in bacteria-containing cells). These findings suggest that Buchnera in the scorpion scorpion may gradually lose its original symbiosis and its function is being replaced by S. symbiotica.

The possible reasons for bacterial substitution are that, due to the bottleneck effect, some bacteria's genome is gradually eroded during vertical propagation, thus gradually losing the original symbiotic ability with the host, so it is easily replaced by bacteria that later invade; or, host insects The living environment has changed. Under the new environmental pressure, some ancient bacteria 
can no longer meet the needs of host metabolism, and the original symbiotic system of the host gradually disintegrates, while some other bacteria gradually form a symbiotic relationship with the host. This replaces the former. In either case, it is possible for the host insect to obtain a more functional bacterial species through substitution, thereby obtaining more and more comprehensive nutrients, thereby facilitating its widening of the living environment or adaptation to changes in environmental conditions. In the case of Nardonella in the elephant, replaced by Sodalis: It is speculated that the original group of the elephants feed on dead wood, but because such foods are difficult to digest and have low levels of nutrients, the early stage of the elephant may rely mainly on some primitive symbiotic bacteria. It supplemented the nutrition; later, the feeding habits of the elephants gradually changed, and some species changed into the feeding of conifer seedlings during the adult stage, and evolved at the same time.

Strong flight ability, able to find a place more suitable for spawning and offspring feeding and development by flying; in this case, the infection of Sodalis bacteria (and thus Nardonella) theoretically helps to improve these mitochondria The level of energy metabolism, so as to meet the needs of its flight, to achieve the purpose of broadening the habitat.

In the existing reports, the cases of bacterial substitution are inferred from the analysis of bacterial community investigation, phylogeny and co-evolutionary relationship. Since the substitution has taken place, it is impossible to find out the specific process of substitution: Whether bacteria directly replace another bacteria through competition, inhibition, etc., or form a stable symbiotic relationship with the host, and then some other mechanisms to promote the original bacteria to lose the evolutionary advantage and then replaced.

A bacterium may inhibit the proliferation and function of other bacteria in the symbiotic system. For example, after infection with Rickettsia, the infection concentration of Buchnera in adult worms decreased by $50 \%$ to $60 \%$ compared with those without Rickettsia, indicating that Rickettsia can significantly inhibit Buchnera. In Drosophila melanogaster, Spiroplasma inhibits the proliferation of Wolbachia, but the latter has no effect on the former. Cardinium was found to induce cytoplasmic incompatibility in both the bee and the whitebacked planthopper, but this function was impaired when Wolbachia was infected, indicating that Wolbachia has a certain inhibitory effect on Cardinium. In general, there are few reports on the inhibition between symbiotic bacteria, and the existing understanding is more fragmentary.

\section{Effects of symbiotic bacteria on insect pathogens}

Many studies have shown that commensal bacteria help to improve the resistance of host insects to a variety of pathogens, including pathogenic bacteria, fungi and viruses. Certain commensal bacteria on the intestinal tract of insects can inhibit the growth of pathogens and protect the host from invasion.

There is little understanding of the physiological and molecular mechanisms of symbiotic bacteria to protect hosts. Studies have shown that when pathogens enter the insect body, some symbiotic bacteria can produce antibacterial substances, or compete with the pathogens, making it difficult for the latter to obtain some essential nutrients, thereby inhibiting the proliferation of pathogens. Direct route. For example, the symbiotic bacteria of D. frontalis of the southern American pine can produce a $20 \mathrm{C}$ carboxylic acid (mycangimycin), which has antibacterial function; the desert gut bacteria can produce antifungal substances such as phenols to suppress Infection with Metarhizium. Indirect pathways are that symbiotic bacteria induce an immune response in the host, which up-regulates the expression of the defense pathway, thereby reducing the host's sensitivity to the pathogen. In addition, from an evolutionary perspective, some species of insects appear to have become dependent on commensal bacteria in resisting pathogens. For example, the genome of pea mites lacks some genes involved in immune responses, and when they are exposed to pathogens, they may rely mainly on commensal bacteria to make up for this "defect." Whether this dependence exists in other insects is unclear. 


\section{Interaction between symbiotic bacteria and symbiotic fungi}

In most insects, symbiotic fungi play a secondary symbiotic role, mainly providing the host with sterols, sugars, amino acids, digestive enzymes, detoxification enzymes and other substances. There are few reports on its relationship with symbiotic bacteria, mainly from Coleoptera, Euphorbiaceae, and other subfamily insects. These insects contain some symbiotic fungi of the genus Cicada, which together with symbiotic bacteria in host food digestion, pheromones It plays an important role in the process of synthesis and resistance to pathogenic bacteria. Studies have found that commensal bacteria may stimulate the growth and reproduction of symbiotic fungi by producing substances such as aromatic metabolites, and may also play the opposite role, ie inhibit the growth of symbiotic fungi. The specific results of the interaction between the two are affected by bacteria and fungi. , host biological characteristics, environmental conditions, certain chemical components in plants (such as $\alpha$-pinene) and other factors. In the future, it is expected to inhibit the function of the latter by interfering with the relationship between commensal bacteria and symbiotic fungi, thereby achieving the purpose of controlling small mites.

\section{Conclusion}

With the deepening of research, people's research on insect symbiotic bacteria gradually evolved from a symbiotic system involving a small number of organisms (such as a bacterium and an insect) to a variety of organisms (including two or more bacteria, one or more related). The symbiotic system of insects is expanding. In the past 10 years, many important advances have been made in the relationship between different symbiotic bacteria and in the relationship between commensal bacteria and other microorganisms in insects. It is particularly noteworthy that based on the analysis of genomic and phylogenetic relationships, it has been basically clear how different bacteria coexisting in the medium and long term of the Homoptera insects together provide some essential nutrients for the host, and which bacteria have been or are in the process of evolution. It has been replaced; at the same time, it has been found that a variety of commensal bacteria have the function of improving the disease resistance of the host insect. These results enrich our understanding of the interactions between different microorganisms in the insect symbiosis system, and provide a scientific basis for the development of new technologies for pest control based on symbiotic relationship regulation. The extensive application of various biological techniques in the fields of entomology, microbiology, and molecular biology has greatly promoted the research on the species, diversity, function, interaction with the host and the co-evolution relationship of insect symbiotic bacteria. It is foreseeable that research in this field will continue to be active in the coming period. In this context, with the increasing number of insects and bacteria being studied, it is expected that more symbiotic systems with greater scientific research value will be found in insects. Especially with the application of various omics techniques and the sequencing of a large number of insects and bacteria, the research on the interaction and substitution of bacteria, the molecular and evolutionary mechanism of bacteria and host interaction is expected to rise to a new height. Based on the research and application of symbiotic bacteria, it is expected that in the near future, new technologies for the biological control of pests such as aphids, whitefly, desert locusts and small mites will be developed.

\section{References}

[1] Baumann P. Biology of bacteriocyte-associated endosymbionts of plant sap-sucking insects [J]. Annual Review of Microbiology, 2005, 59: 155-189.

[2] Oliver K M, Degnan P H, Burke G R, et al. Facultative symbionts in aphids and the horizontal transfer of ecologically important traits [J]. Entomology, 2010, 55: 247-266.

[3] Bordenstein S R. Symbiosis and the origin of species [C]//Bourtzis K, Miller T M, eds. Insect Symbiosis. Boca Raton USA: CRC Press, 2003, 283-304.

[4] Dillon R J, Dillon V M. The gut bacteria of insects: nonpathogenic interactions [J]. Annual Review of Entomology, 2004, 49: 71-92. 